\title{
The Dust Impact on the Intergalactic Medium
}

\author{
L. Montier, M. Giard \\ Centre d'Etudes Spatiales des Rayonnements, 9 avenue Colonel Roche, \\ 31022 Toulouse, France
}

\begin{abstract}
Recent observations at low and high redshift seem to confirm the presence of dust at very low abundances in the InterGalactic Medium (IGM) and especially in the IntraCluster Medium (ICM). We have studied the impact of this dust on the IGM, in terms of heating and cooling. On one hand, with an analytical model of dust emission, we have proved that the dust can be considered as the dominant cooling agent of the ICM at large scale, when the temperature is greater than $T=10^{7} \mathrm{~K}$. On the other hand, with a strong UV Background and a low temperature $\left(T_{\mathrm{e}} \leq 10^{5} \mathrm{~K}\right)$, dust grains become an efficient heating agent of the IGM. These two opposite effects may have played an important role regarding structure formation of the Universe at large and small scales.
\end{abstract}

\section{Introduction}

The presence of dust in the IGM has been first pointed out in extinction by Zwicky (1957) and more recently in emission by Stickel et al. (1998). Naturally all these claims have to be confirmed. However even at a very low abundance, the dust remains one of the important actors of the IGM evolution. The cooling by dust IR emission and the heating by photo-electric effect on dust have been already stressed around SN and QSO (respectively). We have here studied the impact of these two dust effects on the IGM.

\section{The dust emission in the IGM}

The dust grains in the IGM are usually heated by the radiation field and the collisionnal processes, and re-emit this energy by IR emission. However assumming that the dust is placed in a very hot medium far from all luminous sources, the heating function reduces to the electronic collisional heating on a grain given by Dwek \& Werner (1981). In the special case of hot clusters $\left(T \approx 10^{8} \mathrm{~K}\right)$, the dust IR luminosity can be easily compared to the X-ray luminosity given by Mushotzky et al. (1978), and reduces to :

$$
\frac{L_{\mathrm{IR}}^{\mathrm{ICM}}}{L_{\mathrm{X}}^{\mathrm{ICM}}}=0.56\left(\frac{Z_{\mathrm{d}}^{\mathrm{ICM}}}{10^{-5}}\right)\left(\frac{T_{\mathrm{e}}}{10^{8} \mathrm{~K}}\right)^{-1 / 2}
$$

where $Z_{\mathrm{d}}^{\mathrm{ICM}}=M_{\mathrm{d}} / M_{\mathrm{g}}$ is the dust-to-gas mass ratio of the ICM, and $T_{\mathrm{e}}$ is the electronic temperature. 


\section{The dust impact on the IGM}

\subsection{Dust Cooling}

The dust cooling by IR emission has been compared with usual cooling agents such as X-ray bremhstrahlung and atomic processes (like recombination, ionisation by collision, collisionnal excitation). By first computing the ionisation state of the medium, and then the cooling efficiency of the different processes (using the model of Cen 1992) versus the temperature, we have stressed that the dust cooling becomes predominant in the temperature range $10^{6} \mathrm{~K} \leq \mathrm{T} \leq$ $10^{8} \mathrm{~K}$. And smaller is the grain, more efficient becomes the cooling at lower temperature. These conditions are typical of a cooling effect at large scale, accelerating the accretion processes inside the big structures of the universe.

\subsection{Dust Heating}

Inoue \& Kamaya (2003) showed that the heating by photo-electric effect on dust grains (see Weintgartner \& Draine 2001) can be dominant at high redshift over photoionisation for example, and then limit the accretion rate of small strucures. By comparing dust heating and cooling, we have separated their efficiency domain : while dust cooling needs high temperatures and low radiation fields, the dust heating becomes efficient for low temperatures $\left(T_{\mathrm{e}} \leq 10^{5} \mathrm{~K}\right)$ and high UV fields. These two different domains correspond to large and small scale structures of the Universe, respectively.

\subsection{Perspectives}

By accelerating at large scale, and limiting at small scale the accretion rate, the dust could then be considered as one of the actors for structure formation of the Universe. Cosmological simulations are to be performed to confirm or refute these ideas.

\section{References}

Cen, R. 1992, ApJS, 78, 341

Dwek, E., \& Werner, M. W. 1981, ApJ, 248, 138

Inoue, A. K., \& Kamaya, H. 2003, MNRAS, 341, 7

Mushotzky, R. F., Serlemitsos, P. J., Smith, B. W., Boldt, E. A., \& Holt, S. S. 1978, ApJ, 225, 21

Stickel, M., Lemke, D., Mattila, K., Haikala, L.K., \& Haas, M. 1998, A\&A, 329, 55

Weingartner, J. C., Draine, B. T. 2001, ApJS, 134, 263

Zwicky, F. 1957, PASP, 69, 518 